\title{
Physiotherapy-In the Modern Times-The Need vs Necessity
}

\author{
Kanu Kaushik ${ }^{1}$ and Kishore Kumar R ${ }^{2 *}$ \\ ${ }^{1}$ Senior Paediatric Physiotherpaist \& PediaSuit Therapist, Cloudnine Hospital, India \\ ${ }^{2}$ Senior Consultant Neonatologist \& Paediatrician, Cloudnine Hospitals, India
}

*Corresponding author: Kishore Kumar R, Senior Consultant Neonatologist \& Paediatrician, Cloudnine Hospitals, Bangalore \& Adjunct Professor in Neonatology \& Paediatrics, Notre Dame University, Perth, Australia, 1533, 3rd Block Jayanagar, 9th Main, Bangalore - 560011, India

Submission: 眥February 21, 2018 ; Published: 眥 April 17, 2018

\section{Opinion}

Physiotherapy (fizıə(u)' $\theta \varepsilon r ə p i)$ is a noun used to describe traditionally the treatment of disease, injury, or deformity by physical methods such as massage, heat treatment, and exercise rather than by drugs or surgery. In the Cambridge Medical Dictionary, it is described as the treatment of muscle stiffness, pain, and injury by rubbing and moving the sore parts. Physiotherapy is also known as Physical therapy and is one of the allied health professions, is now an emerging and a dynamic profession which is more focused on evidence based studies andby using mechanical force and movements [Bio-mechanics or Kinesiology], Manual therapy, exercise therapy, and electrotherapy, remediates impairments and promotes mobility and function [1-3]. Its extensive clinical application in various health care systems and it helps in preserving, maintaining and promoting optimal physical function of a person. Physical therapy is used to improve a patient's quality of life through examination, diagnosis, prognosis, and physical intervention. It is performed by physical therapists (known as physiotherapists in many countries). Physiotherapists are very well trained to do an assessment, diagnose and rehabilitate any kind of movement dysfunction and disabilities that may be the outcome of any disease, disorders or injuries.

\section{Why do we Need Physiotherapy?}

Our modern lifestyle with all perks and benefits has started showing its results on our body by providing neck \& back strain and knee pain on every day basis and many more on list but this is just not restricted to adults even kids are affected with our modern lifestyle.

Some of the very common lifestyle diseases where physiotherapy is playing a wonderful role in managing them are

\section{Obesity}

India ranks 2nd with obese citizens and numbers are increasing every year according to National Family Health Survey. This is all due to our unhealthy diet \& eating habits, sedimentary lifestyle with lot of stress and reduced physical exercises which all lead to obesity $[4,5]$. People who are obese may suffer from diabetes, cardiovascular diseases, breathing difficulties and variation in blood pressure. Physiotherapy is an ideally placed profession to provide the physical exercises after assessment for weight management and rehabilitation after bariatric surgeries.

\section{Diabetes (Type -2)}

Type II Diabetes is a condition where body fails to produce enough insulin or body is not utilizing it properly. One of the main reasons behind it is obesity. Researchers have proved that physiotherapy exercises are helping people to maintain their insulin level.

\section{Cardiovascular diseases}

This refers to a group of conditions affecting heart, blood vessels and circulatory system. In India, mortality burden is caused maximum by ischemic or coronary heart diseases, Stroke and hypertension. Physiotherapists works on patient's endurance, cardiac output and arterial blood flow by providing aerobic exercises specifically designed according to person's need. Stroke rehabilitation is completely a different segment which is a boon for a patient who is suffered from stroke in all possible aspects [6,7].

\section{Cancer}

It is a condition where body cells grow alarmingly in specific location, reducing body immunity. Cancer rehabilitation is emerging because of cancer survivorship. Therapist works on lymph edema management, pain management, individualized strength training and functional management.

Collaborative Practice is blooming from long time where different health care professionals synchronize together to deliver the highest quality of medical care required to a patient and physiotherapists holds a good position in collaborative practice due to wide spectrum of practice [8]. This practice is very commonly 
seen now days in all multidisciplinary hospitals and health care centers.

Few examples of collaborative practice are as following:

A. A team of orthopedic and physiotherapist in managing musculoskeletal conditions.

B. Neurologist, psychologist, speech therapist, occupational therapist and physiotherapist in managing neurological conditions.

C. Neonatologist, pediatrician, NICU trained nurses, surgeons and pediatric physiotherapist in NICU set up.

D. Cardiovascular surgeons and physiotherapists in managing cardiovascular conditions.

E. Gynecologists and physiotherapist for providing the best care during and after pregnancy.

Awareness in general population regarding physiotherapy is increasing day by day. Now people are coming to physiotherapists as they want to take control of their health and stay well. Some of the areas where physiotherapists assist them are:

A. Cardio respiratory conditions: As a therapist they help in prevention and rehabilitation people affected with heart and pulmonary diseases. Pre \& post surgical rehabilitation is improving quality of life.

B. Women's health: Physiotherapists helps during pregnancy phase, post-partum care, breast feeding, pelvic floor dysfunctions, and loss of bladder and bowl control.

C. Geriatric health care: They are providing evidence based therapies to manage or prevent age related problems like osteoporosis, arthritis, frequent falling because of poor balance, urine incontinence.

D. Pediatric care: Pediatric physiotherapists are providing care from NICU till the kids are grown up. Pre terms babies, milestone delay children, cerebral palsy, autism and many other pediatric conditions are taken care efficiently [9].

E. Orthopedic conditions: They help in prevention or managing acute or chronic musculoskeletal disorders like neck and back pain, sports injuries, post surgical management after fractures, TKR, etc.

F. Neurological Conditions: Patients with severe brain or spinal cords damage are seeing major change after following up with physiotherapy exercises. Physiotherapists are able to improve quality of life in patients who are suffering from stroke, Parkinson's disease and many other neurological conditions.

G. Sports management: Sports physiotherapists are in high demand as they help in prevention, diagnosing and treating sports injuries on field.

Aquatic therapy is a ray of hope for many patients now as a therapist can treat and rehabilitate many conditions in a pool like paraplegic patients strengthening program, stroke rehabilitation exercises for balancing and coordination, knee strengthening exercises for osteoarthritis patients, kids with neurological conditions like cerebral palsy, retts syndrome, down syndrome and even in geriatric rehabilitation also. It's Normal to Change over the Years, Sudden Increase in Physiotherapy Demand Is Because Of Preferences, Opinions and Beliefs.

\section{References}

1. American Physical Therapy Association (1997) Guide to Physical Therapist Practice. Physical Therapy 77(11): 1168-1650.

2. Groven KS, Heggen K (2018) Physiotherapists encounters with obese patients: Exploring how embodied approaches gain significance. Phsyiother Thoery Pract 34(5): 346-358.

3. Bonnevie T, Gravier FE, Debeaumont D, Viacroze C, Muir JF, et al. (2018) Home based neuromuscular electrical stimulation as an add-on to pulmonary rehabilitation does not provide further benefits in patients with chronic obstructive pulmonary disease. A multicenter randomized trial. Arch Phys Med Rehabil pii: S0003-9993(18)30100-X.

4. (1946) World Health Organization. Preamble to the Constitution of the World Health Organization as adopted by the International Health Conference, New York, USA, pp. 19-22.

5. (2011) World Confederation for Physical Therapy. WCPT guideline for the development of a system of legislation/regulation/recognition of physical therapists. London, UK.

6. (2001) American Physical Therapy Association, Guide to Physical Therapist Practice. In: American Physical Therapy Association ( $\left.2^{\text {nd }} e d n\right)$, 81(1): 9-744.

7. Cott CA, Finch E, Gasner D, Yoshida K, Thomas SG, et al. (1995) The Movement Continuum of Physical Therapy. Physiotherapy Canada 47(2): 87-95.

8. Higgs J, Jones M (1995) Clinical Reasoning in the Health Professions. Higgs J, Jones M (Eds.), Clinical Reasoning in the Health Professions. Oxford, Butterworth-Heinemann, UK.

9. (1996) Standing Liaison Committee of Physiotherapists in the European Union. In: Standing Liaison Committee of Physiotherapists in the European Union. The Practice of Physiotherapy in the European Union. Brussels, Belgium: SLCP, UK. 
(C) (i) Creative Commons Attribution 4.0 International License

For possible submissions Click Here
EPMR Examines in
Physical Medicine
\& Rehabilitation

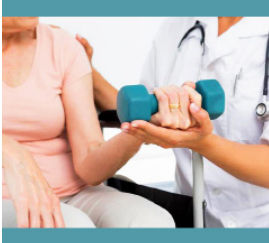

Examines in Physical Medicine and Rehabilitation: Open Access

\section{Benefits of Publishing with us}

- High-level peer review and editorial services

- Freely accessible online immediately upon publication

- Authors retain the copyright to their work

- Licensing it under a Creative Commons license

- Visibility through different online platforms 